\title{
Clinical Application of T-SPOT.TB Using Pleural Effusion as a Diagnostic Method for Tuberculosis Infection
}

\author{
Yoshihiro Kobashi, Keiji Mouri, Yasushi Obase, Shigeki Kato, Mikio Oka \\ Department of Respiratory Medicine, Kawasaki Medical School, Kurashiki, Japan \\ Email: voshihiro@med.kawasaki-m.ac.jp
}

Received 4 February 2014; revised 4 March 2014; accepted 11 March 2014

Copyright $@ 2014$ by authors and Scientific Research Publishing Inc.

This work is licensed under the Creative Commons Attribution International License (CC BY). http://creativecommons.org/licenses/by/4.0/

\section{Open Access}

\begin{abstract}
Introduction: The objective of this study was the comparison of the results of T-SPOT.TB using pleural effusion (PE) with those of IGRAs using peripheral blood (PB) or other diagnostic methods for the diagnosis of tuberculous (TB) pleurisy. Methods: We measured adenosine deaminase (ADA) in PE, QuantiFERON TB-Gold In-Tube (QFT), and T-SPOT.TB using PB, and T-SPOT.TB using PE. The definite group of TB pleurisy included 12 patients and other disease group 33 patients. Main findings: Sensitivity for QFT using PB was $83 \%$ and specificity was $85 \%$, sensitivity for T-SPOT.TB using PB was $92 \%$ and specificity was $82 \%$, while sensitivity for ADA in PE was $83 \%$ and specificity was $76 \%$. When we adopted the same cut-off level of a positive response for T-SPOT.TB as PB using PE, sensitivity for T-SPOT.TB using PE was $100 \%$ and specificity was $82 \%$, respectively. Although there were no significant differences among the four diagnostic methods, sensitivity for T-SPOT.TB using PE gave the most accurate diagnosis of TB-definite patients compared to ADA in PE or QFT using PB. Conclusions: If we performed T-SPOT.TB using a local specimen from the infection site, we could obtain a higher sensitivity than IGRAs using PB or ADA in PE and the numbers of ESAT-6 and CFP-10-positive SFCs were 3 to 5 fold higher in PEMCs than in PBMCs. T-SPOT.TB using PE may become a useful diagnostic method for TB pleurisy.
\end{abstract}

\section{Keywords}

T-SPOT.TB, QuantifERON, Adenosine Deaminase, Pleural Effusion (PE), Peripheral Blood (PB)

\section{Introduction}

Tuberculosis (TB) is a major cause of morbidity and mortality worldwide [1]. TB pleurisy is the second most

How to cite this paper: Kobashi, Y., et al. (2014) Clinical Application of T-SPOT.TB Using Pleural Effusion as a Diagnostic Method for Tuberculosis Infection. Open Journal of Respiratory Diseases, 4, 64-72. 
common extrapulmonary manifestation of Mycobacterium tuberculosis (MTB) infection following TB lymphangitis, accounting for up to $23 \%$ of all extrapulmonary TB patients [2].

The positive rate of smear and culture examinations for MTB, or the detection rate of MTB-DNA from pleural effusion (PE), ranges from 12\% - 70\%, and [3] 0\% - 100\%, respectively [3]-[6]. Pleural biopsy for culture of MTB, MTB nucleic acid amplification and histological detection of caseating epitheloid granuloma showed sensitivities of $40 \%-80 \%, 90 \%$, and $50 \%-97 \%$, respectively [7]-[10]. Although the procedure is invasive and is associated with more clinical complications, the sensitivity to detect active TB disease is higher in biopsies compared with PE. The diagnosis of TB pleurisy is difficult to establish, not only in terms of the nonspecific clinical presentation, but also due to the lack of useful diagnostic examinations.

In interferon-gamma release assays (IGRAs), there are two in vitro tests which measure the interferon-gamma release of activated T cells isolated from the patient's peripheral blood; Quanti FERON-TB (QFT) as an enzymelinked immunosorbent assay (ELISA) and T-SPOT.TB as an enzyme-linked immunospot assay (ELISPOT). Of the two IGRAs, T-SPOT.TB has a higher sensitivity and is applicable to other clinical specimens excluding peripheral blood as a supporting diagnostic method for active TB disease because it measures IFN- $\gamma$-producing $\mathrm{T}$ cells after regulation of the mononuclear cell count (the cell count of pleural effusion mononuclear cells (PEMCs) was $2.5 \times 10^{5}$, the same as peripheral blood mononuclear cells (PBMCs)).

Therefore, we evaluated the results of T-SPOT.TB using PE including the usual IGRAs results using peripheral blood without invasive diagnostic methods to diagnose TB pleurisy in routine clinical practice.

\section{Methods}

\subsection{Patients}

We prospectively evaluated 45 patients with lymphocyte-dominant exudative pleural effusion. These clinical specimens were collected from Kawasaki Medical School Hospital (Kurashiki, Japan) and five affiliated hospitals and the final diagnosis was decided between January 2009 and September 2013. This study was approved by the ethics committee of Kawasaki Medical School, and written informed consent was obtained from all subjects.

Peripheral blood (PB) was obtained by drawing venous blood for MTB-specific ELISA (QuantiFERON TB-Gold In-Tube (QFT)) and MTB-specific ELISPOT (T-SPOT.TB) before the initiation of antituberculous treatment. PE were also extracted before the initiation of antituberculous treatment and microbiological examinations for acid-fast bacilli, MTB-specific DNA amplification, ADA and MTB-specific ELISPOT were performed. These samples were firstly examined by Ziehl-Neelsen staining and were digested and decontaminated by the sodium hydroxide method. They were inoculated onto slants of 1\% Ogawa egg medium (Japan BCG, Tokyo, Japan) and identified by growth characteristics and conventional biochemical tests. Mycobacteirium tuberculosis was identified by an Amplicor polymerase chain reaction assay (Roche Diagnostic Systems, Branchburg, NJ, USA) and also identified by DNA-DNA hybridization (DDH) methods (Kyokuto Pharmaceuticals, Tokyo, Japan).

\subsection{Definitions of Patients in the TB-Definite Group and the Other Disease Group}

We firstly decided the TB definite group patients if MTB was cultured from PE or a pleural biopsy specimen. Secondly, we decided the other disease group patients if other clinical diagnosis was established by the detection of malignant cells or satisfying the diagnostic criteria of other disease. We excluded patients with healed pulmonary TB from the other disease group patients to avoid the influence on the results of T-SPOT.TB when the specificity was calculated in this study.

\subsection{ADA in PE}

The ADA activity in PE was measured by an auto analyzer using commercially available kits (Nittobo Medical, Tokyo, Japan). The cut-off level of ADA activity in PE was judged as a positive result at over $36 \mathrm{U} / \mathrm{ml}$ according to the study of Ogata et al. [11].

\subsection{QFT Test}

QFT was performed according to the instructions of the manufacturer (Cellestis Ltd). The judgment was performed according to the guidelines proposed by the Centers for Disease Control and Prevention (CDC) [12]. 


\subsection{T-SPOT.TB}

MTB-specific ELISPOT assays were made using the T-SPOT.TB test [13] [14]. PBMCs and PEMCs were prepared from peripheral blood (PB) and $\mathrm{PE}$ which obtained before the initiation of antituberculous treatment. In brief, $2.5 \times 10^{5}$ PBMCs and $2.5 \times 10^{5}$ PEMCs were placed overnight on 96-well plates that had been pre-coated with a mouse anti-human IFN- $\gamma$ antibody in $100 \mu$ volumes of culture medium per well. The cells were left unstimulated (negative control), or were stimulated with $50 \mu$ of phytohaemagglutinin (positive control) or $50 \mu l$ of ESAT-6 and CFP-10 peptides in separate wells. The culture of the plates, washing, counterstaining, visualization and analysis of the spots were performed according to the manufacturer's instructions. The response of stimulated cultures was considered positive when the test well contained six spots or above and had twice the number of spots shown in the control well. The background number of spots in negative control wells (nil wells) was below 10 spots per well in both PBMCs and PEMCs of all patients.

\subsection{Statistical Analysis}

All statistical analyses were performed using Stat View software Version 6 (Artec Institute, Osaka, Japan). The ADA, IFN- $\gamma$, and spot forming cell (SFC) levels in the two patient groups are expressed as the median level. Mann-Whitney tests were used to compare the differences between two groups. Sensitivity was calculated by dividing the number of positive results tested into the number of patients in the TB-definite group for all clinical practice. Specificity was calculated by dividing the number of negative results tested into the number of patients in the other disease group, excluding patients with healed tuberculosis, for all clinical practice. A probability value of less than 0.05 was considered significant.

\section{Results}

The TB-definite group for TB pleurisy consisted of 12 patients and the other disease group consisted of 33 patients (lung cancer in 24 patients, asbestos-related pleural disease in 5, collagen vascular disease in 3, malignant lymphoma in 1). Clinical findings of these cases showed lymphocyte-predominant exudative PE are shown in Table 1. Concerning the backgrounds of the two patient groups, the frequency of respiratory diseases in the underlying diseases was significantly higher than those in the other disease group. There were no significant differences among the two patient groups in the laboratory data.

The median levels of ADA in PE and the positive response rates were significantly higher in the TB-definite group (positive response rate: 83\%, median level: $66.6 \mathrm{IU} / \mathrm{l}$ ) than the other disease group (positive response rate: 21\%: median level: 20.9 IU/l), as shown in Figure 1. The sensitivity of ADA in PE was 83\% and the specificity was $79 \%$, respectively.

Regarding the results of QFT using PB, while the positive response rate was 83\% in the TB-definite group, it was significantly lower in the other disease group (9\%). On the other hand, indeterminate results accounted for $8 \%$ in the TB-definite group and 6\% in the other disease group (Table 2). Indeterminate results of both groups caused by low response of mitogen (positive control) in all patients. The sensitivity of QFT was 83\% and the specificity was $85 \%$, respectively.

As for the results of T-SPOT.TB using PB and PE, while the positive response rate was 92\% in the TB-definite group and $15 \%$ in the other disease group by using peripheral blood mononuclear cells (PBMC), it was $100 \%$ in the TB definite group and 18\% in the other disease group using pleural effusion mononuclear cells (PEMC) when we used the same cut-off values (6 spot forming cells (SFCs)), as well as PBMC (Table 3 and Table 4). Also, while the sensitivity of PBMC was $92 \%$ and the specificity of PBMC was $82 \%$, the sensitivity of PEMC was $100 \%$ and the specificity was $82 \%$, respectively.

The median numbers of SFCs in the two groups were significantly higher in patients in the TB-definite group (PBMC: median level 18 SFCs versus PEMC: median level 83 SFCs) than the other disease group (PBMC: median level 2 SFCs versus PEMC: median level 3 SFCs) for the T-SPOT.TB results using PBMC and PEMC (Figure 2 and Figure 3). Especially, the median numbers of SFCs of T-SPOT.TB using PEMC were significantly higher than those of T-SPOT.TB using PBMC in the TB-definite group.

\section{Discussion}

IGRAs such as QFT or T-SPOT.TB have recently become popular as supportive diagnostic methods for TB 


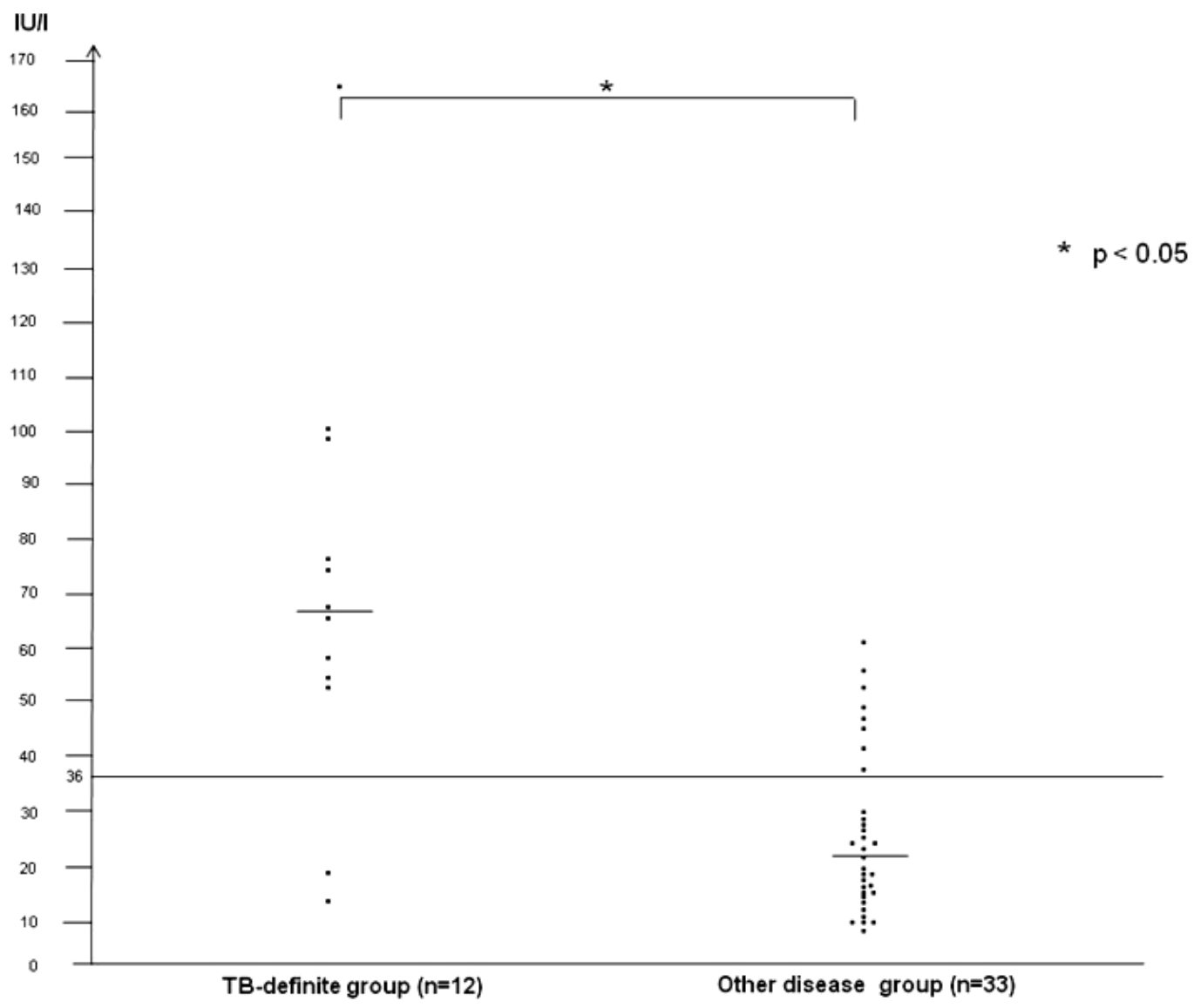

Figure 1. ADA (Pleural effusion).

Table 1. Clinical findings of cases that showed lymphocyte-predominant exudative pleural effusion.

\begin{tabular}{|c|c|c|}
\hline & $\begin{array}{l}\text { TB-definite group } \\
\qquad(\mathrm{n}=12)\end{array}$ & $\begin{array}{l}\text { Other disease group } \\
\qquad(\mathrm{n}=33)\end{array}$ \\
\hline Age (Median \pm S.D.) & $75.5 \pm 19.8$ & $72.0 \pm 10.9$ \\
\hline Gender (Male: Female) & $8: 4$ & $23: 10$ \\
\hline Smoking history & $8(67 \%)$ & $23(70 \%)$ \\
\hline Underlying disease & 9 (83\%) & $33(100 \%)$ \\
\hline Respiratory disease & $4^{*}(33 \%)$ & $29^{*}(88 \%)^{\#}$ \\
\hline Non-respiratory disease & $8^{*}(67 \%)$ & $22^{*}(67 \%)$ \\
\hline Immunosuppressive treatment & $1(8 \%)$ & $4(12 \%)$ \\
\hline \multicolumn{3}{|l|}{ Laboratory data } \\
\hline \multicolumn{3}{|l|}{ Peripheral blood } \\
\hline WBC (cells/ $\mu \mathrm{l})$ & $5040 \pm 2150$ & $5370 \pm 550$ \\
\hline Lymphocyte (cells/ $\mu \mathrm{l})$ & $995 \pm 555$ & $1100 \pm 106$ \\
\hline Total protein (g/dl) & $6.9 \pm 0.6$ & $6.9 \pm 0.6$ \\
\hline Albumin (g/dl) & $3.3 \pm 0.5$ & $3.2 \pm 0.5$ \\
\hline
\end{tabular}

*Duplication (+), N.S.: Not significant. ${ }^{*} \mathrm{p}<0.05$. 
Table 2. QFT (Peripheral blood).

\begin{tabular}{|c|c|c|}
\hline $\begin{array}{l}\text { Clinical diagnosis } \\
\text { QFT }\end{array}$ & $\begin{array}{l}\text { TB-definite group } \\
\qquad(\mathrm{n}=12)\end{array}$ & $\begin{array}{l}\text { Other disease group } \\
\qquad(\mathrm{n}=33)\end{array}$ \\
\hline Determinate & $11(92 \%)$ & 31 (94\%) \\
\hline \multirow[t]{2}{*}{ Positive } & 10 (83\%) & \multirow[t]{2}{*}{$3(9 \%)$} \\
\hline & & \\
\hline Negative & $1(8 \%)$ & 28 (85\%) \\
\hline Indeterminate & $1(8 \%)$ & $2(6 \%)$ \\
\hline
\end{tabular}

${ }^{*} \mathrm{p}<0.05$.

Table 3. T-SPOT.TB (PBMC).

\begin{tabular}{|c|c|c|}
\hline $\begin{array}{l}\text { Clinical diagnosis } \\
\text { T-SPOT.TB }\end{array}$ & $\begin{array}{l}\text { TB-definite group } \\
\qquad(\mathrm{n}=12)\end{array}$ & $\begin{array}{l}\text { Other disease group } \\
\qquad(\mathrm{n}=33)\end{array}$ \\
\hline Determinate & 12 (100\%) & 32 (97\%) \\
\hline Positive & 11 (92\%) & 5 (15\%) \\
\hline Negative & $1(8 \%)$ & $27(82 \%)$ \\
\hline Indeterminate & $0(0 \%)$ & $1(3 \%)$ \\
\hline
\end{tabular}

PBMC: Peripheral blood mononuclear cell. ${ }^{*} \mathrm{p}<0.05$.

Table 4. T-SPOT.TB (PEMC).

\begin{tabular}{|c|c|c|}
\hline $\begin{array}{l}\text { Clinical diagnosis } \\
\text { T-SPOT.TB }\end{array}$ & $\begin{array}{l}\text { TB-definite group } \\
\qquad(\mathrm{n}=12)\end{array}$ & $\begin{array}{l}\text { Other disease group } \\
\qquad(\mathrm{n}=33)\end{array}$ \\
\hline Determinate & 12 (100\%) & 33 (100\%) \\
\hline Positive & $\begin{array}{c}12(100 \%) \\
\mathrm{L}\end{array}$ & $\begin{array}{c}6(18 \%) \\
-1\end{array}$ \\
\hline Negative & $\begin{array}{c}0(0 \%) \\
\llcorner\end{array}$ & $\begin{array}{l}27(82 \%) \\
ـ\end{array}$ \\
\hline Indeterminate & $0(0 \%)$ & $0(0 \%)$ \\
\hline
\end{tabular}

PEMC: Pleural effusion mononuclear cell. ${ }^{*} \mathrm{p}<0.05$.

disease. They have a high sensitivity of 83\% - 97\% [15] [16], and a high specificity of 92\% - 100\% [16] [17]. However, IGRAs cannot distinguish between active TB disease and latent TB infection (LTBI) or healed TB disease using PB. The reason is that only a minority of lymphocytes are found in PB [18]. Moreover, the number of MTB-specific T cells with regard to ESAT-6 and CFP-10 specific T-cells is very low (0.002\% - 0.4\%), even in active TB disease [19] [20]. On the other hand, antigen-specific T-cells migrate to the site of inflammation in active TB disease and rapidly release some cytokines such as interferon-gamma (IFN- $\gamma$ ) [21] [22]. While an ELISA assay such as QFT quantifies IFN- $\gamma$ production following MTB-specific antigen contact in 


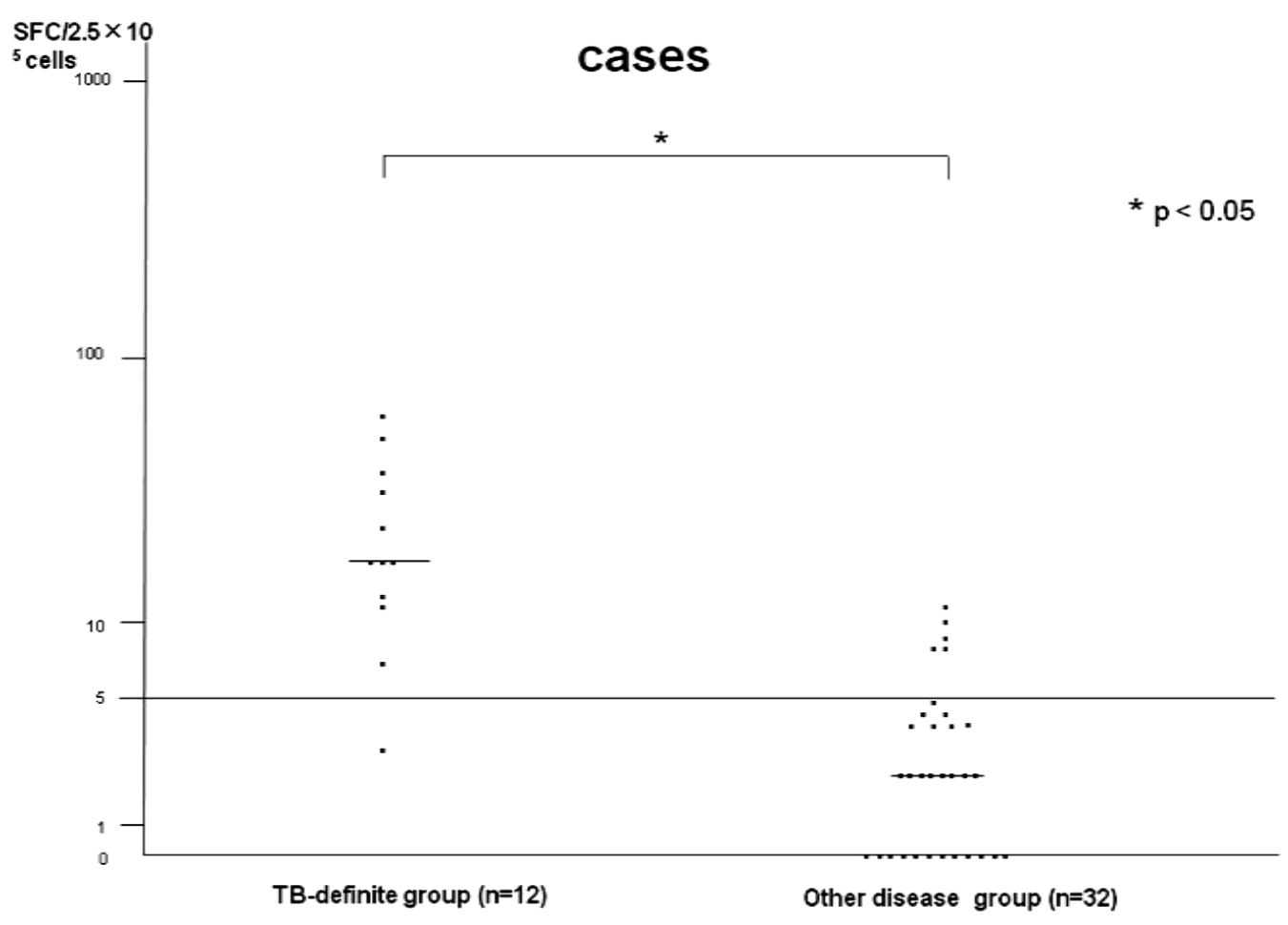

Figure 2. T-SPOT.TB (PBMC) determinate cases.

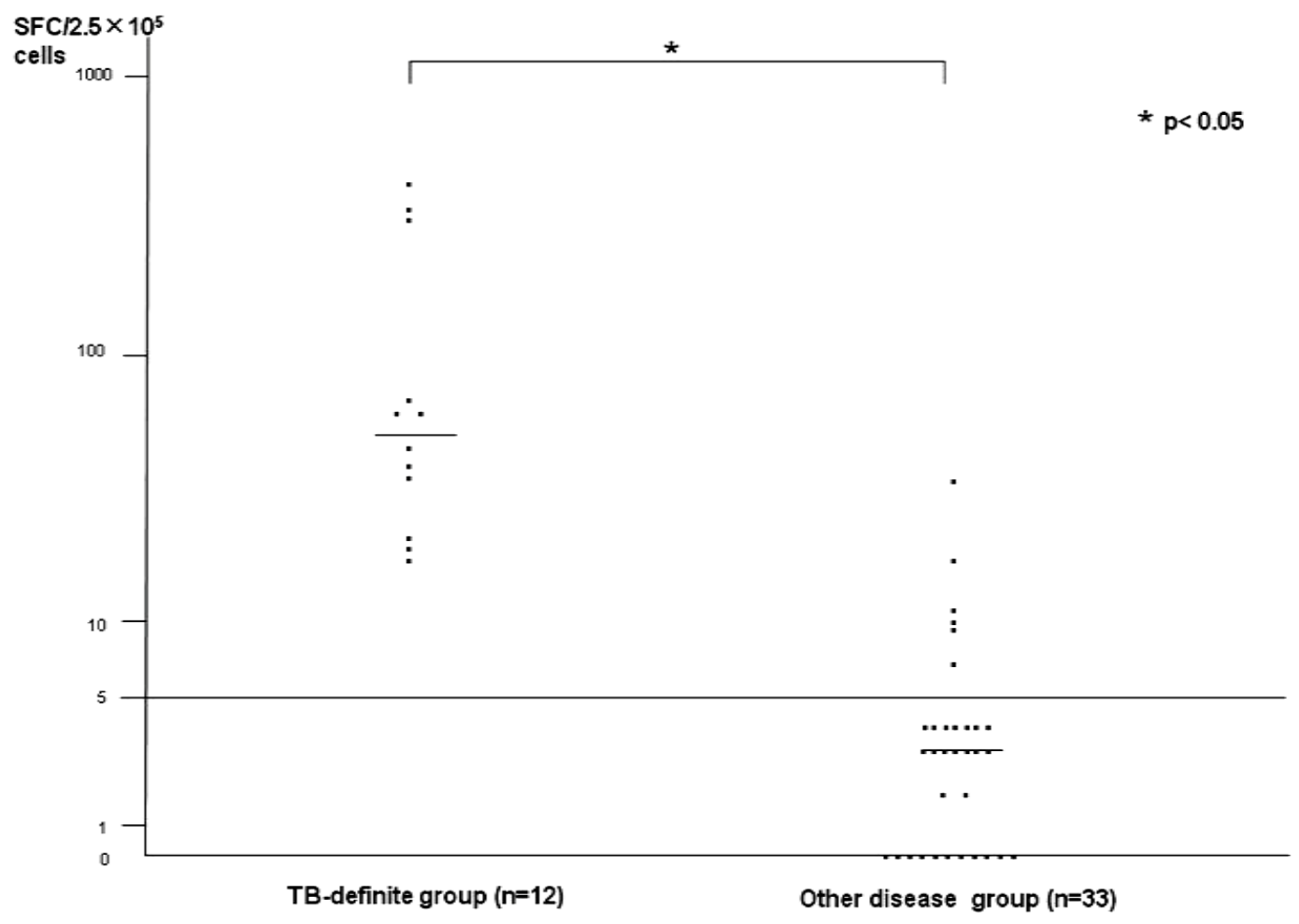

Figure 3. T-SPOT.TB (PEMC).

clinical specimens, it is possible to enumerate single MTB-specific cells using an ELISPOT assay such as T-SPOT.TB. Therefore, because ELISPOT assay results can compensate for lymphocyte numbers $\left(2.5 \times 10^{5}\right)$ in 
order to compare the results of T-SPOT.TB using PBMC and PEMC, we measured MTB-specific cells in not only PB, but also PE using an ELISPOT assay in this study.

Regarding the study of TB pleurisy, a first report indicated that the median number of ESAT-6 SFCs in PEMCs was 15-fold higher than that in PBMCs [23]. Losi et al. reported that the diagnostic sensitivity of T-SPOT.TB for active tuberculous pleurisy was very high (95\%) by enumerating antigen-specific mononuclear cells from PE of patients with exudative pleurisy, but the specificity was only 76\% [24]. The median concentrations of ESAT-6 and CFP-10-specific cells were 4.2 to 7.5 fold higher in PEMCs than in PBMCs. In this study, although total number of patients tested was low because of intermediate incidence of TB disease such as Japan, we confirmed excellent results for the diagnostic sensitivity of T-SPOT.TB using PE for active TB pleurisy in combination with patients in the TB-definite group (100\%) compared to the results of ADA (83\%) in PE, QFT (83\%) and ELISPOT assays using PBMC (92\%), while the median numbers of ESAT-6 and CFP-10-positive SFCs were 3 to 5 fold higher in PEMCs than in PBMCs.

On the other hand, when we judged the positive response of PEMC at the same cut-off level as PBMC ( $\geq 6$ SFCs), the diagnostic specificity for the ELISPOT assays using PEMC showed almost same percentage (82\%) (not significant) for ADA (76\%) and QFT (85\%). There were six patients who showed false-positive responses for the ELISPOT assay in the other disease group; four patients with carcinomatous pleuritis, one with pleuritis combined with collagen vascular disease, and one with malignant lymphoma. It was reported that the sensitivity was generally higher in the ELISPOT assay (T-SPOT.TB) compared to the ELISA assay (QFT), and the specificity was better in QFT than T-SPOT.TB [25] [26]. It is unknown why six patients showed false-positive responses for T-SPOT.TB using PE through this study. Therefore, we have to try to answer the question in the ongoing study. Indeterminate results of QFT and T-SPOT.TB were detected in one case in the TB-definite group and two patients in the other disease group due to low response of mitogen (positive control). These patients had severe underlying disease or collagen vascular disease, were receiving immunosuppressive treatment and showed lymphocytopenia and/or hypoalbuminemia on laboratory findings. Although we reported that lymphocytopenia and/or hypoalbuminemia may cause indeterminate IGRAs results in previous reports [27] [28], these findings support our hypothesis.

There are some limitations involved in the use of IGRAs in PE samples. Firstly, although the cut-off level of T-SPOT.TB using PBMC is defined, the cut-off levels for IGRAs using PE have not yet been defined. We challenged the estimation of ROC curve analysis or AUC calculation to ascertain the appropriate cut-off levels for ESAT-6 and/or CFP-10 for PEMC in this study, but because the numbers of patients were small scale in individual group, we could not obtain the appropriate cut-off levels for PEMC. Although several reports have used various cut-off levels derived from small scale studies in heterogeneous settings [24], this causes some problems in routine clinical application. A large, comparative study is required to determine the appropriate cut-off levels for positivity, and this may be different in countries with a high or low incidence of TB disease. Secondly, when we calculated the specificity in this study, we used patients who showed lymphocyte-dominant exudative pleural effusion due to other underlying disease except healed pulmonary TB as a negative control group. However, there is no evidence which can completely exclude this influence on the results of IGRAs. Regarding the specificity of IGRAs, although age-wise analysis may be required to evaluate previous TB infection, we could not do age-wise analysis regarding specificity because there were not many patients (33 patients) in the other disease group and most of these patients were old in this study. In retrospect, we think that we need perform a large scale study including many younger age patients. Thirdly, the total numbers of patients with TB-definite group enrolled in this study was low because of intermediate incidence of TB disease such as Japan. Therefore, the results may be considered preliminary and need to be confirmed in large scale cohorts, including immunocompromised patients such as HIV-positive patients, and in countries with a higher incidence of TB disease [29].

\section{Conclusion}

The diagnosis of TB pleurisy is difficult in clinical practice using standard diagnostic methods. In particular, MTB-NAT in PE or PCE cultures has low diagnostic sensitivity. Therefore, methods for establishing a definite diagnosis are desirable. For such cases, when thoracocentesis is technically feasible, although there are no assessment criteria for ELISPOT assay for MTB using PE, a strong positive ELISPOT assay for MTB using PE (very high SFCs of PEMCs compared to PBMCs were recognized in this study) may be a rapid test before the initiation of antituberculous treatment. The further large scale investigation is needed to assess both the sensitivity 
and specificity of the ELISPOT assay for MTB using PE applied to the diagnosis of TB pleurisy.

\section{Acknowledgements}

The authors would like to thank T. Matsushima (Kurashiki Daiichi Hospital, Kurashiki, Japan, Asahigaoka Hospital, Okayama, Japan), N. Okimoto (Kawasaki Medical School Kawasaki Hospital, Okayama, Japan), Masaaki Abe (Takahashi Central Hospital, Takahashi, Japan) and J. Nakamura, H. Yoneyama (Kasaoka Daiichi Hospital, Kasaoka, Japan) for their constructive comments.

\section{Funding}

This work was supported by MEXT KAKENHI (23591168).

\section{References}

[1] Elzinga, G., Raviglione, M.C. and Maher, D. (2004) Scale up: Meeting Targets in Global Tuberculosis Control. Lancet, 363, 814-819. http://dx.doi.org/10.1016/S0140-6736(04)15698-5

[2] Vidal, R., De Gracia, J., Ruiz, J., et al. (1986) Controlled Study of 637 Patients with Tuberculosis. Diagnosis and Therapeutic Results with 9- and 6-Month Regimens. Medical Clinics (Barcelona), 87, 368-370.

[3] Gopi, A., Madhaven, S.M., Sharma, S.K., et al. (2007) Diagnosing and Treatment of Tuberculous Pleural Effusion in 2006. Chest, 131, 880-889. http://dx.doi.org/10.1378/chest.06-2063

[4] Valdes, L., Alvarez, D., San Jose, E., et al. (1998) Tuberculous Pleurisy: A Study of 254 Patients. Archieves of Internal Medicine, 158, 2017-2021. http://dx.doi.org/10.1001/archinte.158.18.2017

[5] Aggarwal, A.N., Gupta, D. and Jindal, S.K. (1999) Diagnosis of Tuberculous Pleural Effusion. Indian Journal of Chest Disease Allied Science, 41, 89-100.

[6] Nagesh, B.S., Sehgal, S., Jindal, S.K., et al. (2001) Evaluation of Polymerase Chain Reaction for Detection of Mycobacterium tuberculosis in Pleural Fluid. Chest, 119, 1737-1741. http://dx.doi.org/10.1378/chest.119.6.1737

[7] Prakash, U.B. and Reiman, H.M. (1985) Comparison of Needle Biopsy with Cytologic Analysis for the Evaluation of Pleural Effucison: Analysis of 414 Cases. Mayo Clinic Proceedings, 60, 158-164. http://dx.doi.org/10.1016/S0025-6196(12)60212-2

[8] Seibert, A.F., Haynes Jr., J., Middleton, R., et al. (1991) Tuberculous Pleural Effusion. Twenty-Year Experience. Chest, 99, 883-886. http://dx.doi.org/10.1378/chest.99.4.883

[9] Epstein, D.M., Kline, L.R., Albelda, S.M., et al. (1987) Tuberculous Pleural Effusions. Chest, 91, 106-109. http://dx.doi.org/10.1378/chest.91.1.106

[10] Escudero Bueno, C., Garca Clemente, M., Vuesta Castro, B., et al. (1990) Cytologic and Bacteriologic Analysis of Fluid and Pleural Biopsy Specimens with Cope’s Needle. Study of 414 Patients. Archieves of Internal Medicine, 150, 1190-1194. http://dx.doi.org/10.1001/archinte.150.6.1190

[11] Ogata, Y., Aoe, K., Hiraki, A., Murakami, K., Kishino, D., Chikamori, K., Maeda, T., Ueoka, H., Kiura, K. and Tanimoto, M. (2011) Is Adenosine Deaminase in Pleural Fluid a Useful Marker for Differentiating Tuberculosis from Lung Cancer or Mesothelioma in Japan, a Country with Intermediate Incidence of Tuberculosis? Acta Medica Okayama, 65, 259-263.

[12] Mazurek, G.H., Jereb, J., Vernon, A., et al. (2010) Updated Guidelines for Suing Interferon Gamma Release Assays to Detect Mycobacterium tuberculosis Infection-United States, 2010. MMWR Recommendations and Reports, 59, 1-25.

[13] Lalvani, A., Pathan, A.A., McShane, H., et al. (2001) Rapid Detection of Mycobacterium Tuberculosis Infection by Enumeration of Antigen-Specific T Cells. American Journal of Respiratory and Critical Care Medicine, 163, 824-828. http://dx.doi.org/10.1164/ajrccm.163.4.2009100

[14] Lalvani, A., Magvenkar, P., Udwadia, Z., et al. (2001) Enumeration of T Cells Specific for RD1-Encoded Antigens Suggests a High Prevalence of Latent Mycobacterium tuberculosis Infection in Healthy Urban Indians. Journal of Infectious Disease, 183, 469-477. http://dx.doi.org/10.1086/318081

[15] Richeldi, L. (2006) An Update on the Diagnosis of Tuberculosis Infection. American Journal of Respiratory and Critical Care Medicine, 174, 736-742. http://dx.doi.org/10.1164/rccm.200509-1516PP

[16] Menzies, D., Pai, M. and Comstock, G. (2007) Meta-Analysis: Nee Tests for the Diagnosis of Latent Tuberculosis Infection: Areas of Uncertainly and Recommendations for Research. Annals of Internal Medicine, 146, 340-354. http://dx.doi.org/10.7326/0003-4819-146-5-200703060-00006

[17] Yew, W.W. and Leung, C.C. (2007) Update in Tuberculosis 2006. American Journal of Respiratory and Critical Care 
Medicine, 175, 541-546. http://dx.doi.org/10.1164/rccm.200611-1653UP

[18] Westermann, J. and Bode, U. (1999) Distribution of Activated T Cells Migrating through the Body: A Matter of Life or Death. Immunology Today, 20, 302-306. http://dx.doi.org/10.1016/S0167-5699(99)01474-7

[19] Wilkinson, K.A., Wilkinson, R.J., Pathan, A., et al. (2005) Ex Vivo Characterization of Early Secretory Antigenic Target 6-Specific T Cells at Sites of Active Disease in Pleural Tuberculosis. Clinical Infectious Disease, 40, 184-187. http://dx.doi.org/10.1086/426139

[20] Jafari, C., Ernst, M., Kalsdorf, B., et al. (2006) Rapid Diagnosis of Smear-Negative Tuberculosis by Bronchoalveolar Lavage Enzyme-Linked Immunospot. American Journal of Respiratory and Critical Care Medicine, 174, 1048-1054. http://dx.doi.org/10.1164/rccm.200604-465OC

[21] Okamoto, M., Hasegawa, Y., Hara, T., et al. (2005) T-Helper Type 1/T-Helper Type 2 Balance in Malignant Pleural Effusions Compared to Tuberculous Pleural Effusion. Chest, 128, 4030-4035. http://dx.doi.org/10.1378/chest.128.6.4030

[22] Dieli, F., Friscia, G., Sano, C., et al. (1999) Sequestration of T Lymphocytes to Body Fluids in Tuberculosis: Reversal of Anergy Following Chemotherapy. Journal of Infectious Disease, 180, 225-228. http://dx.doi.org/10.1086/314852

[23] Hirsch, C.S., Toossi, Z., Johnson, H., et al. (2001) Augmentation of Apotosis and Interferon- $\gamma$ Production at Sites of Active Mycobacterium Tuberculosis Infection in Human Tuberculosis. Journal of Infectious Disease, 183, 779-788. http://dx.doi.org/10.1086/318817

[24] Losi, M., Bossink, A., Codecase, L., et al. (2007) Use of a T-Cell Interferon- $\gamma$ Release Assay for the Diagnosis of Tuberculous Pleurisy. European Respiratory Journal, 30, 1173-1179. http://dx.doi.org/10.1183/09031936.00067307

[25] Higuchi, K., Kawabe, Y., Mitarai, S., et al. (2009) Comparison of Performance in Two Diagnostic Methods for Tuberculosis Infection. Medical Microbiology and Immunology, 198, 33-37. http://dx.doi.org/10.1007/s00430-008-0102-5

[26] Diel, R., Loddenkemper, R. and Nienhaus, A. (2007) Evidence-Based Comparison of Commercial Interferon- $\gamma$ Release Assays for Detecting Active TB: A Metaanalysis. Chest, 137, 952-968. http://dx.doi.org/10.1378/chest.09-2350

[27] Kobashi, Y., Mouri, K., Obase, Y., et al. (2007) Clinical Evaluation of QuantiFERON TB-2G Test for Immunocompromised Patients. European Respiratory Journal, 30, 945-950. http://dx.doi.org/10.1183/09031936.00040007

[28] Kobashi, Y., Sugiu, K., Mouri, K., et al. (2009) Indeterminate Results of QuantiFERON TB-2G Test Performed in Routine Clinical Practice. European Respiratory Journal, 33, 812-815. http://dx.doi.org/10.1183/09031936.00075008

[29] Lange, C., Hellmich, B., Ernst, M., et al. (2007) Rapid Immunodiagnosis of Tuberculois in a Woman Receiving AntiTNF Therapy. Nature Clinical Practice of Rheumatology, 3, 528-534. http://dx.doi.org/10.1038/ncprheum0571 